\title{
Astrocyte-Synapse Receptor Coupling in Tripartite Synapses: A Mechanism for Self-Observing Robots
}

\author{
Bernhard J. Mitterauer \\ Volitronics-Institute for Basic Research, Psychopathology and Brain Philosophy, University of Salzburg, Salzburg, Austria \\ Email: mitterauer.b@gmail.com
}

How to cite this paper: Mitterauer, B.J. (2018) Astrocyte-Synapse Receptor Coupling in Tripartite Synapses: A Mechanism for Self-Observing Robots. Advances in Bioscience and Biotechnology, 9, 63-82. https://doi.org/10.4236/abb.2018.92006

Received: January 16, 2018

Accepted: February 10, 2018

Published: February 14, 2018

Copyright $\odot 2018$ by author and Scientific Research Publishing Inc. This work is licensed under the Creative Commons Attribution International License (CC BY 4.0).

http://creativecommons.org/licenses/by/4.0/

\section{(c) (i) Open Access}

\begin{abstract}
A model of an intentional self-observing system is proposed based on the structure and functions of astrocyte-synapse interactions in tripartite synapses. Astrocyte-synapse interactions are cyclically organized and operate via feedforward and feedback mechanisms, formally described by proemial counting. Synaptic, extrasynaptic and astrocyte receptors are interpreted as places with the same or different quality of information processing described by the combinatorics of tritograms. It is hypothesized that receptors on the astrocytic membrane may embody intentional programs that select corresponding synaptic and extrasynaptic receptors for the formation of receptorreceptor complexes. Basically, the act of self-observation is generated if the actual environmental information is appropriate to the intended observation processed by receptor-receptor complexes. This mechanism is implemented for a robot brain enabling the robot to experience environmental information as "its own". It is suggested that this mechanism enables the robot to generate matches and mismatches between intended observations and the observations in the environment, based on the cyclic organization of the mechanism. In exploring an unknown environment the robot may stepwise construct an observation space, stored in memory, commanded and controlled by the intentional self-observing system. Finally, the role of self-observation in machine consciousness is shortly discussed.
\end{abstract}

\section{Keywords}

Tripartite Synapse, Cyclic Organization, Receptor Coupling, Self-Observation, Conscious Robots 


\section{Introduction and Hypothetical Model}

Neurons and glia are the two major classes of cells in the brain. Their main difference lies in their electrical excitability [1]. Neurons are electrically excitable, whereas glia are not [2]. Macroglia consist of astrocytes and oligodendrocytes. Here I focus on astrocytes that build units of interaction with the neuronal synapse, called tripartite synapse [3] [4]. The model proposed here deals with astrocyte-synapse interactions and the formation of receptor-receptor complexes that may represent a central mechanism for self-observation and be implemented in robot brains.

Whereas brain-oriented approaches to machine consciousness are mainly based on the neuronal system [5], my model concentrates on the glial system of the brain. Currently, robot consciousness [6] is an emerging field that addresses the problem of designing and implementing computational models of consciousness in a robot [7] [8] [9] [10]. Models of self-observation in learning [11] and machine vision [12] have been significantly developed in the last two decades [13].

The hypothetical model of self-observing systems is the following: in tripartite synapses various receptor types are located on the presynapse, postsynapse, extrasynaptically, and on the astrocytic membrane. Neurotransmitters (NT) activate astrocytic receptors leading to an increase of $\mathrm{Ca}^{2+}$ concentration that stimulates the production of gliotransmitters (GT). I hypothesize that the coupling of homo- (composed of the same receptor type) and hetero- (composed of a different receptor type) receptor complexes in tripartite synapses [14] is commanded and controlled by GT. This mechanism is cyclically organized and operates by the formalism of proemial counting [15] based on the interchange between feedforward and feedback operations. Receptors are interpreted as places with the same or different quality or domain of synaptic information processing, formally described as tritograms [16] [17]. Decisively, receptor patterns on the astrocyte may embody intentional programs that via GT select the corresponding receptor types forming receptor-receptor complexes.

Based on this synaptic model an intentional self-observing system is proposed which operates on the two place systems of the astrocytic receptors and the neuronal receptors. The astrocytic receptors represent the pattern of intended observations and the neuronal receptors the pattern of the environmental observations. Observed domains (qualities) that correspond to the intended domains are stored in memory, non-corresponding domains are rejected. The match/mismatch pattern, stored in memory, represents a space of observations. After $n$ steps the robot has generated a pattern of positions of intended domains of observation in the explored environment.

The study starts out with the biological background of structure and function of astrocyte-synapse interactions in tripartite synapses. Then the cyclic organization of synaptic information processing is described according to the formalism of proemial counting. Furthermore, the formation of synaptic receptor-receptor 
complexes is outlined and formally interpreted by the combinatorics of tritograms. Based on the hypothesis that astrocytic receptors embody intentional programs of observation selecting environmental information from the neuronal receptor complexes, the implementation of an intentional self-observing system is proposed. Finally, the problem of machine consciousness is shortly discussed.

\section{Perisynaptic Astrocytic Processes}

Primarily five to eight processes per cell emanate from the cell body of protoplasmic astrocytes [18]. They subdivide progressively to form finer and finer processes of up to 100.000 per astrocyte. Most of these processes interact with synapses, termed perisynaptic astrocytic processes (PSAPs) [19]. Fewer processes contact vessels, called perivascular astrocytic processes (PVAPs) [20]. Some processes (an average of 11) contact other astrocytes forming gap junctions and an astroglial network [20]. Importantly, PSAPs extend and withdraw from synapses in an activity dependent manner [21].

Figure 1 outlines the elementary structure of PSAPs. Stem processes emanating from the soma of the astrocyte arborize and form PSAPs (and PVAPs). Synapses are located on the fine end processes and ensheathed by contacting and retracting from synapses. PSAPs express G-protein coupled receptors capable of sensing neurotransmitters, increasing cytosolic calcium levels, and activating the release of gliotransmitters interacting with receptors on the presynapse, postsynapse, and extrasynaptically. In addition, PSAPs contribute to the maintenance of ion homeostasis, for example by regulating extracellular $\mathrm{K}^{+}$concentrations, and $\mathrm{pH}[22]$.

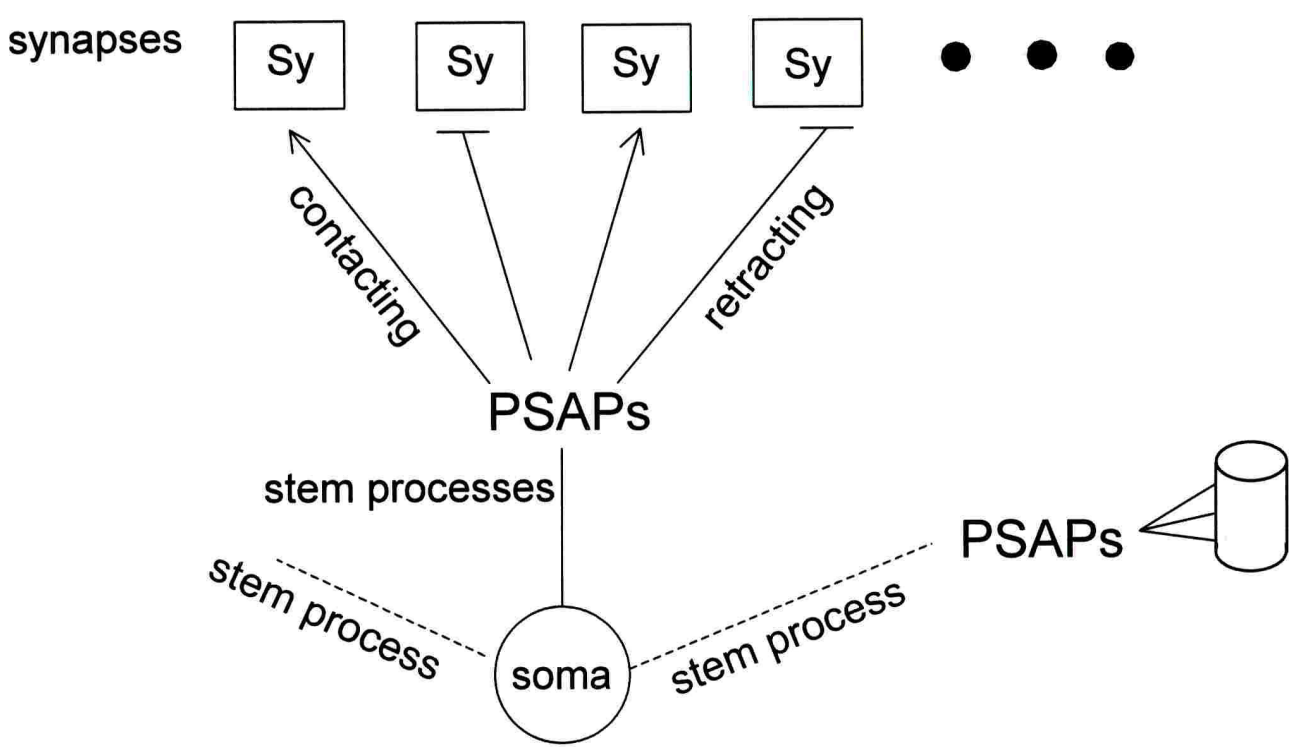

From the soma of the astrocyte stem processes emanate and arborize into perisynaptic astrocytic processes (PSAPs). These endprocesses contact $(\rightarrow)$ or retract $(\vdash)$ from synapses. In addition, astrocytic processes contact vessels, called perivascular astrocytic processes (PVAPs) (only one is shown here).

Figure 1. Schematic representation of perisynaptic astrocytic processes (PSAPs) and perivascular astrocytic processes (PVAPs). 


\section{Tripartite Synapses}

It is experimentally well established that signaling between neurons and astrocytes runs bidirectionally [23]. Astrocytes can be triggered by synaptic activity through activation of neurotransmitter receptors on astrocytes elevating $\mathrm{Ca}^{2+}$ concentrations that stimulate the release of neuroactive substances, called gliotransmitters. Gliotransmitters (e.g. glutamate, adenosine-tri-phosphate, D-serine) modulate synaptic excitability and synaptic transmission [24]. These synapseastrocyte interactions lead to the new concept of the tripartite synapse [3].

Figure 2 shows a schematic representation of a glutamatergic tripartite synapse. The excitatory neurotransmitter glutamate (GLU) released from the presynapse activates cognate postsynaptic (por), extrasynaptic (esr), and astrocytic receptors (acr). GLU is uptaken by transporters ( $\mathrm{t}$ ). The occupancy of acr by GLU elevates the $\mathrm{Ca}^{2+}$ concentration which stimulates the production of gliotransmitters (GT). GT feeds back to the cognate receptors on the presynapse (psr). Since astrocytes express most of the receptors identified in neurons [25], they are able to sense and respond to neuronal signals and modulate synaptic transmission [26].

Mathematical modeling of synaptic plasticity demonstrated that astrocytes modify the synaptic flow of information through neuronal networks and create structural changes in synapses. Based on physiological or biophysical experiments mathematical modeling and computer simulations will probably be helpful for testing predictions on functions of astrocytes in neuronal networks [27]. Such simulations may also give rise to new ideas concerning the role of astrocytes in

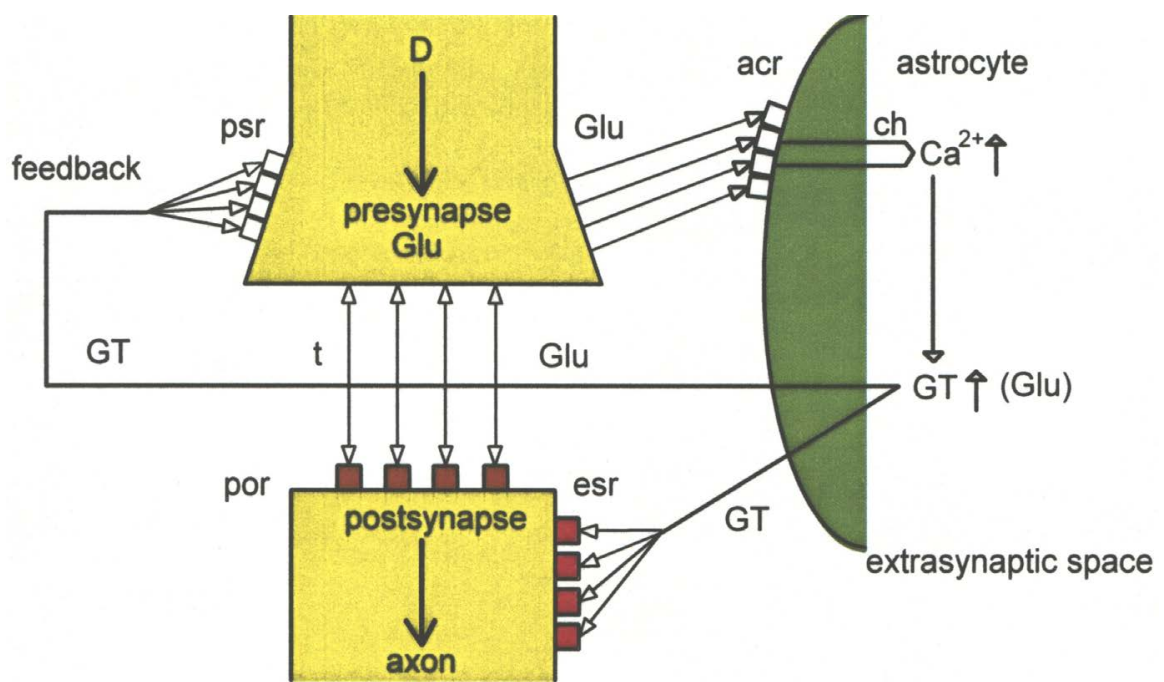

The excitatory neurotransmitter glutamate (GLU) is activated by a dendrite (D). GLU activates postsynaptic receptors (por) and is reuptaken on the presynapse via transporters ( $t$ ). GLU also occupies receptors on the astrocyte activating channels (ch) that leads to an increase in calcium concentration and to the production of gliotransmitters (GT). The release of GT from the astrocyte occupies presynaptic receptors (psr), por and extrasynaptic receptors on the postsynapse. The effect of GLU corresponds with a feedback mechanism on the presynapse and the depolarization by the occupancies of postsynaptic and extrasynaptic receptors.

Figure 2. Schematic diagram of a glutamatergic synapse. 
explaining the complexity of the brain [28] [29] [30]. In their biophysical model Volman and coworkers [31] demonstrated the coupling between synaptic transmission and the local calcium concentration on an enveloping astrocyte domain. Due to this interaction the astrocyte regulates the information flow from presynaptic to postsynaptic cells depending on previous activity at this and other nearby synapses, comparable to a gatekeeping effect. Nadkarni and coworkers [32] using a computer model showed that astrocytes optimize the synaptic transmission. In this approach the dynamics of the intra-astrocyte $\mathrm{Ca}^{2+}$ level is decisive. Accordingly, astrocytes respond to synaptic activity via intracellular $\mathrm{Ca}^{2+}$ dynamics, which in turn feeds back to neurons by triggering the release of GT [23].

Synaptic activity of low frequency leading to local astrocyte $\mathrm{Ca}^{2+}$ signals probably triggers localized gliotransmission restricted to exerting feedback modulation of the active synapse [33]. In the case of increased synaptic activity, astrocyte $\mathrm{Ca}^{2+}$ signals may fill the whole astrocyte resulting in gliotransmission that exerts feedforward actions on the synapse [34]. Indeed, it is experimentally well established that tripartite synapses operate on feedforward and feedback loops [35] [36].

\section{Gliotransmission}

In the perspective of the proposed model gliotransmitters (GT) may play a significant role in the formation of synaptic and extrasynaptic receptor-receptor complexes. GT are chemical transmitter substances released from astrocytes that modulate glial-neuronal interactions in tripartite synapses [37]. The capability of astrocytes to induce excitability with various $\mathrm{Ca}^{2+}$ concentrations enables the production of GT. The function of GT depends on the type of GT, each occupying cognate synaptic and extrasynaptic receptors [38]. The major types of GT released from the astrocytes are glutamate (GLU), adenosine-tri-phosphate (ATP) and D-serine.

GLU is not only an excitatory neurotransmitter but it can also function as a GT because of its ability to increase $\mathrm{Ca}^{2+}$ concentrations in astrocytes [39]. GLU is tightly filled into synapse-like vesicles released from astrocytes dependent on an elevation of $\mathrm{Ca}^{2+}$ concentration. The main receptors activated by GLU are kainate receptors, metabotrophic GLU receptors, and N-methyl-D-asparate (NMDA) receptors [37]. Several release mechanisms play a role in gliotransmission [40]. There are reverse operations of glutamate transporters on the plasma membrane; opening of anion transporters induced by cell-swelling; purine $2 \times 7$ receptors release GLU; gap junction hemichannels on the astrocytic cell membrane also release GLU.

Basically, ATP suppresses synaptic transmission. ATP released from astrocytes activates $\mathrm{Ca}^{2+}$ waves enabling the communication between astrocytes. Presently, it is not fully understood if gliotransmission mediated by ATP is calciumdependent or not. However, experimental evidence indicates that ATP release is 
partly dependent on $\mathrm{Ca}^{2+}$ and SNARE proteins including multiple pathways based on exocytosis [39].

Although the transmitters mediating astrocyte-synapse signaling are mainly believed to be GLU [41] or ATP [39], it is clear that the amino acid D-serine also functions as an important gliotransmitter. D-serine is synthesized in astrocytes and released by GLU receptor activation through $\mathrm{Ca}^{2+}$ and SNARE-dependent exocytosis [42]. Van Horn and coworkers [43] proposed a model of D-serine transmission in tripartite synapses. Accordingly, activation of the presynapse results in release of GLU which binds to a-amino-3-hydroxy-5-methyl-4-isoxazoleproprionic acid receptors (AMPAR) on neighboring astrocytes causing the release of D-serine that binds to synaptic N-methyl-D-asparate (NMDA) containing GLUN2A subunits. Basically, all gliotransmitters identified may exert a modulatory function on synaptic transmission by the activation of cognate neuronal receptors.

\section{Cyclic Organization of Astrocyte-Synapse Communication}

As already pointed out, it is well established experimentally that astrocytes communicate with neurons in a bidirectional manner [44] in tripartite synapses operating on feedforward and feedback loops [23] [35] [36]. DePitta and colleagues [23] elucidated how astrocyte-synapse interactions significantly differ from conventional synapses. In the latter information processing occurs exclusively via one input and one output function. Moreover, the three components of the tripartite synapse process information in loops. Here, two input functions and two output functions operate unidirectionally generating loops [23].

The proemial relationship introduced by Guenther [45] provides a formal notion of "no unidirectionality". The proemial relationship is based on directional relations that include both unidirectional "ordered" and bidirectional "exchange" operations. Given is a relator $x$ with a directional relation $(\rightarrow)$ with a relatumy. If the relatum becomes a relator, the exchange is not mutual, but in a higher logical order according to the following formalism:

If $R_{i+1}\left(x_{i} ; y_{i}\right)$ is given and the relatum ( $x$ or $y$ ) becomes a relator, the notation is $\mathrm{R}\left(\mathrm{x}_{\mathrm{i}-1} ; \mathrm{y}_{\mathrm{i}-1)}\right.$ where $\mathrm{R}_{\mathrm{i}}=\mathrm{x}_{\mathrm{i}}$ or $\mathrm{y}_{\mathrm{i}}$. Reversely, if the relator becomes a relatum, $\mathrm{R}_{\mathrm{i}+2}$ $\left(\mathrm{x}_{\mathrm{i}+1} ; \mathrm{y}_{\mathrm{i}+1}\right)$ is obtained with $\mathrm{R}_{\mathrm{i}+1}=\mathrm{x}_{\mathrm{i}+1}$ or $\mathrm{y}_{\mathrm{i}+1}$. Here, the subscription $i$ indicates a higher or lower logical order. The rationale of a proemial relationship is that if the relator becomes a relatum, a relationship is established that operates not in the former logical order, but in a higher one. An atom is a representative example of what the variables $\left(\mathrm{x}_{\mathrm{i}} ; \mathrm{y}_{\mathrm{i}}\right)$ mean.

If we consider an atom as a relation between more elementary particles, then the elementary particles assume the part of the relata. But the atom is a relatum in a more complex order within a molecule. Therefore, an atom is both, relative to the elementary particles it is a relator, but it can change this property with the one of the relatum if we consider it within the more comprehensive relationship of a molecule (45). 


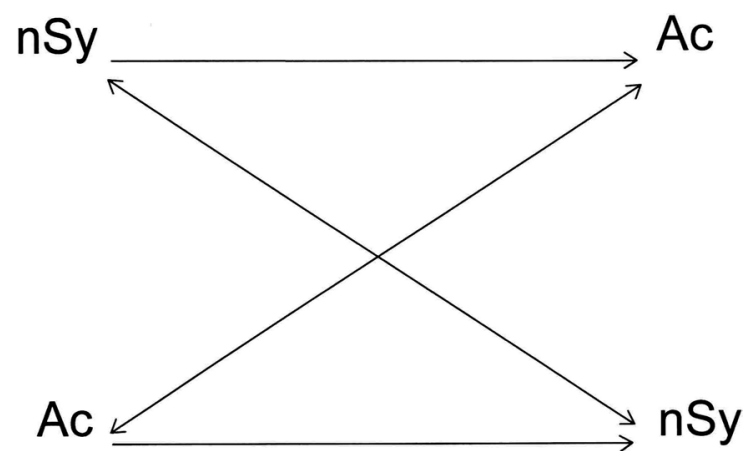

The neuronal synapse (nSy) and the astrocyte (Ac) function both as a relator and as a relatum. It is shown that if nSy is directionally connected with the astrocyte, nSy functions as the relator and Ac as the relatum. Then the relation changes $(\leftrightarrow)$ so that Ac functions as the relator and nSy as the relatum. These operations are based on two directional $(\rightarrow)$ and two bidirectional $(\leftrightarrow)$ relations generating a cyclic system of synapse-astrocyte interactions.

Figure 3. Cyclic interaction between the neuronal synapse and the astrocyte.

Since astrocyte-synapse interactions run in loops, it is additionally necessary to describe a cyclic proemial relationship as a closed system where directional and bidirectional relations interchange. Applying the loop generation in tripartite synapses, the cyclic proemial relationship is drawn in Figure 3. The neuronal synapse (nSy) functions as a relator and the astrocyte (Ac) as a relatum $(\rightarrow)$. If this directional relation changes, Ac becomes a relator and nSy a relatum. This relationship is based on two directional relations $(\rightarrow)$ and two bidirectional relations $(\leftrightarrow)$ representing a cyclic system of interactions.

In addition, if we interpret a system of relators and relata on numbers, the system can be proemially counted [15]. The normal concept of counting starts at one point and ends on another. In contrast to this arithmetical counting, proemial counting counts both directional "ordered" and bidirectional "exchange" operations. Since self-observation may be based on cyclic processes, the model proposed operates on the proemial relationship or counting [15].

\section{Model of Self-Observation}

Baer [46] stated that a theory of observation is a prerequisite for a better understanding of quantum mechanics in brain science. Whereas cyclic operations in tripartite synapses may enable self-reflection [15], self-observation may additionally require special mechanisms that compare activated neuronal receptors with astrocytic receptors by receptor-receptor coupling.

\section{Receptor-Receptor Coupling in Wiring and Volume Transmission}

Volume and synaptic transmission are interrelated by the ability of their chemical signals to activate a great variety of receptor promotors in heteroreceptor complexes located in the plasma membrane, either synaptically or extrasynaptically [47]. The extracellular space is organized in a highly organized extracellular matrix (ECM) whose molecules build synaptic and perisynaptic scaffolds. These scaffolds determine the clustering of neurotransmitter receptors in the postsy- 
naptic compartment presenting barriers that reduce the lateral diffusion of membrane proteins away from synapses, contributing to functional compartmentalization in the brain [48]. Importantly, astrocytes may play a key role in the organization of ECM.

Fuxe and coworkers [49] describe the integration of synaptic and volume transmission signals as receptor-receptor interactions in heteroreceptor complexes in pre- and postsynaptic membranes and their signaling cascades and also in extrasynaptic homoreceptor and heteroreceptor complexes, a fact which may influence the responsiveness of neurons without changing the synaptic responses [47]. Excitingly, volume transmission and receptor-receptor interactions in the catecholamine/oxytocin neuron interaction in the brain may control social behavior and pair bonding [49]. Basically, allosteric receptor-receptor interactions generate a high diversity and bias which also produces selectivity. Diversity is in part achieved by the dynamic formation of heteroreceptor complexes, each equipped with multiple functions through the allosteric receptor-receptor interactions responsible for synaptic modification in the brain circuits. Selection can be achieved by a choice of the optimal signaling responses from multiple heteroreceptor complexes in the single neuron, enhancing synaptic efficacy in the selected network [50]. Importantly, the reorganization of hetero-homoreceptor complexes through a formation change or disappearance of receptor complexes can stabilize the pattern of transmitter release [47].

\section{Astrocytic Receptor Complexes May Embody Intentional Programs}

Basically, receptors for the common receptor type are identified on the astrocytic membrane [25]. Most importantly, in a mathematical model Tarakanov and Fuxe [51] computed 48 pairs of receptor-receptor interactions which form receptor heteromers, others build non-heteromers. The latter type of receptor complexes may operate in the intentional self-observing system proposed here (section 9).

Intentional actions are mainly investigated in neuronal networks [52]. For example, Proekt and coworkers [53] showed in a simple neuronal network a dynamical basis of intentions and expectations. Basically, if one gives reasons for consciousness, intentionality must be taken into account [54]. Intentionality is here defined as an intentional program generating a specific multi-relational structure in the inner and outer environment controlled by the specific program principle [55]. I hypothesize that receptor complexes on the astrocyte may embody intentional programs that are coupled with G-protein coupled receptors (GPCRs) which are capable of modulating neuronal excitability and firing patterns in a new way through changes in neuronal receptor function [56]. Most recent, Flock and colleagues [57] provided insight into constraints underlying selective coupling, focusing on GPCRs. If we consider the realization of intentional programs, a selection mechanism must basically operate. 
Tarakanov and Fuxe [51] formally described how prototriplets of amino acid residues and their "teams" may be applied to construct a kind of code selecting specific receptors responsible for forming heterodimers. Based on the obtained results a "guide- and clasp" manner for receptor-receptor interactions is described, where "adhesive guides" might be the triplet homologies [51]. In the perspective of the present study, the generation of astrocytic receptor complexes represents a "guide pattern" for the "clasping" of neuronal signals operating as an intentional program. Here we may deal with an elementary mechanism of information structuring as shown in visual perception [58].

\section{Model of Synaptic Receptor-Receptor Coupling by Gliotransmitters}

I hypothesize that the formation of receptor-receptor complexes in tripartite synapses may be determined by gliotransmitters (GT), as outlined in Figure 4. Given the neurotransmitters (NT) glutamate (GLU), serotonine (SE), and noradrenaline (NA), both the synaptic receptors and the cognate astrocytic receptors (acR) are activated by GLU and SE, but not by NA. GT are produced by acR activation by NT increasing $\mathrm{Ca}^{2+}$ concentration. GT feed back to the cognate synaptic and extrasynaptic receptors forming homoreceptor (of the same receptor type) and heteroreceptor (of different receptor types) complexes. In Figure 4 the homoreceptor complexes GLU-GLU, SE-SE, and the heterocomplexes GLU-SE, GLU-G-protein coupled-SE are depicted.

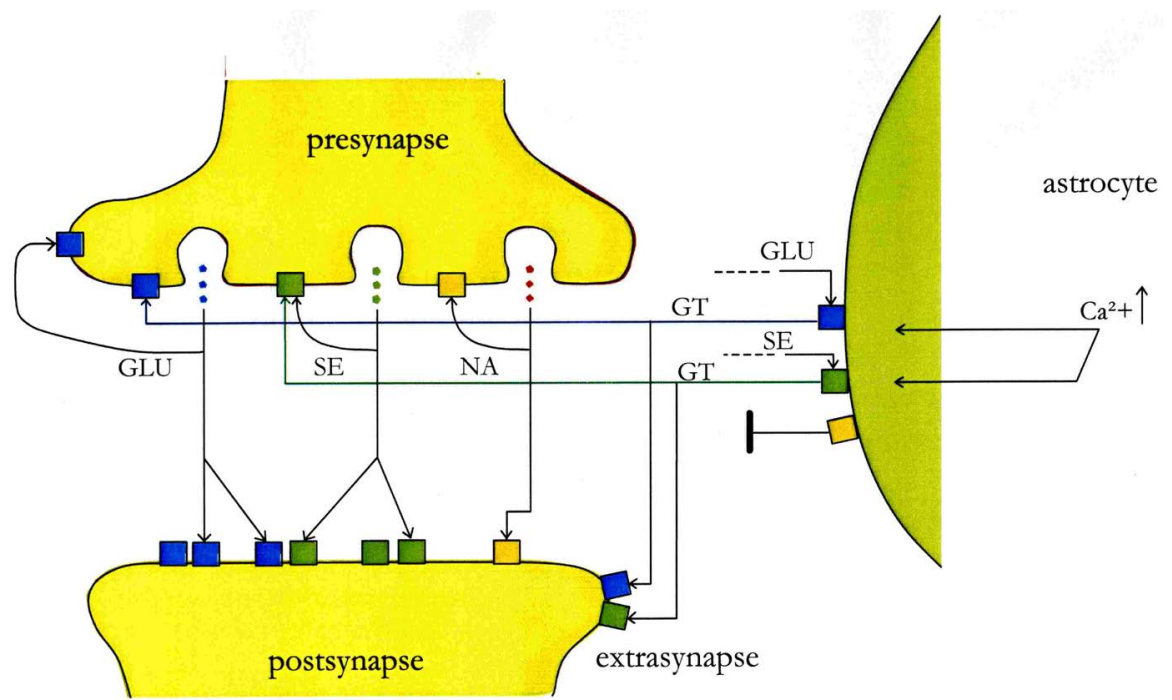

Vesicles (v) of the presynapse release the neurotransmitters glutamate (GLU, blue), serotonine (SE, green), and noradrenaline (NA, orange). These transmitters form the homoreceptor complexes GLU-GLU, SE-SE, and the heterocomplexes GLU-SE. A G-protein coupled GLU-GLU is exemplified. GLU and SE occupy cognate receptors on the astrocyte that elevates $\mathrm{Ca}^{2+}$ concentrations ( $\uparrow$ ) stimulating the production of gliotransmitters (GT). GT for GLU and SE feed back to the cognate presynaptic receptors (psr). Since the astrocytic receptors for NA are not activated, no feedback occurs to the psr. Hence, GT select the receptors for synaptic and extrasynaptic receptor-receptor coupling.

Figure 4. Schematic diagram of receptor-receptor coupling determined by gliotransmitters. 
Decisively, NA does not activate cognate acRs so that no feedback in synaptic receptors occurs. Consequently, receptor-receptor complexes of the NA-type cannot be generated. Therefore, gliotransmission may select specific receptor types for the formation of receptor-receptor complexes. In my perspective receptor patterns on the astrocytes may embody intentional programs selecting and composing the domains (qualities) of synaptic information processing.

\section{Intentional Self-Observing System}

Generally, self-observation analyzes environmental information by means of experience-based intentional programs with commanding and controlling function in preferred locations of the brain. The generation of receptor-receptor complexes in wired and volume transmission may provide a brain-biological model for the implementation of a novel intentional self-observing system. Basically, a synaptic or extrasynaptic receptor sheath is composed of $n$ receptors that embody the same or different qualities of information processing. Receptors on the astrocyte may function as intentional programs ready for the activation by appropriate transmitter substances. The fundamental principle of the implementation of self-observation is as follows: if a brain is capable of self-observation, the concept of intention to observe something and the concept of the observed must be located in different places. The selection mechanism of the intended observation operates on the comparison between the activation patterns of the two-place systems and becomes stepwise reflected by proemial counting. It should be mentioned that receptor-receptor coupling is mainly a theoretical model [51], interpreted here as an elementary mechanism of self-observation.

Receptors can formally be described as tritograms [28] that represent a categorization of receptor qualities. The combinatorics of tritograms has been introduced by Guenther [16] and applied to brain models by the present author [28] [59]. The generation of a tritogrammatic structure follows the Bell numbers. Bell numbers count the number of partitions of a set. The sequence of Bell numbers is $1,2,5,52,203,877 \ldots$ For example, the tritostructure of three different tritograms is generated by the combinatorics of all possible positions of places with the same or different quality. Importantly, since the position of places in each tritogram is decisive, we are dealing with qualitative counting of different receptor categories. Table 1 shows the combinatorics of three different tritograms generating the tritostructure of five tritograms corresponding to the Bell number 5. Regarding the biological model proposed, each tritogram represents the receptor pattern of five perisynaptic astrocytic processes.

In the brain computing occurs in categories or domains, a fact which is experimentally supported with regard to the visual cortex [59]. Accordingly, by means of a category-specific biasing mechanism in object-selective cortex categorial information in natural scenes can be rapidly detected. Objects which belong to the target category are processed up to the category level, even when presented outside the focus of attention. On the contrary, objects not belonging 
Table 1. Tritostructure of 5 tritograms corresponding to 5 astrocytic receptor complexes on astrocytic processes.

\begin{tabular}{cccccc}
\hline & & \multicolumn{3}{c}{ receptor complexes } \\
\hline \multirow{2}{*}{ astrocytic process } & $\mathrm{a}$ & $\mathrm{a}$ & $\mathrm{a}$ & $\mathrm{a}$ & $\mathrm{a}$ \\
& $\mathrm{a}$ & $\mathrm{a}$ & $\mathrm{b}$ & $\mathrm{b}$ & $\mathrm{b}$ \\
tritograms & $\mathrm{a}$ & $\mathrm{b}$ & $\mathrm{a}$ & $\mathrm{b}$ & $\mathrm{c}$ \\
& 1 & 2 & 3 & 4 & 5 \\
\hline
\end{tabular}

Each tritogram consists of the same or different places symbolized by the small letters a, b, and c. Since the position is relevant, one can also speak of qualitative counting of different domains. This tritostructure is interpreted as the formal basis of an astrocyte with 5 processes, each embodying a receptor sheath with qualitatively different receptor complexes.

to the relevant category are not represented at the category level, even if presented inside the focus of attention [60]. In my present model, the proposed intentional programming selects relevant domains or categories of observation and rejects irrelevant domains (see section 6). Here, we may deal with an elementary mechanism of information processing in the brain.

\section{Implementation of the Self-Observing System}

The self-observing system consists of two receptor complexes that compute on tritograms. One receptor complex embodies programs which intend to observe $n$ domains in the environment. The other receptor complex computes domains observed in the environment via visual perception systems. The self-observing system operates as follows: if the intentional receptor complexes on the astrocyte are activated, they become coupled with the activation pattern of the receptor complexes of visual perception. On each place the intended domain of observation is compared with the observed domain in the environment. The robot is stepwise moving through an unknown environment. When all intended domains of observation are compared with the observed domains, the robot moves on for testing the next receptor complex by comparison with the observed domains. If the intended domain does not correspond to the observed domain, a mismatch is given.

As described above, each counting step forward must also be counted back, generating cycles so that the system is basically cyclically organized and therefore self-reflexive. Figure 5 gives a simple example of a double cycle-place structure generating a match/mismatch memory of intended domains of observation in the environment. Three receptor places $(a, b, c)$ build five receptor complexes. If the intended domain corresponds to the observed domain, the comparison is designated by a double headed arrow in the sense of a match. In the case of non-correspondence (cross), a mismatch results between intention and observation. In the first receptor complex the quality [a] is intended and corresponds to [a'] observed in the environment. By counting back from the observed domain to the intended domain, a cycle is generated. On the next position [a] intends the observation quality [a] again, but the observed quality [b'] is 


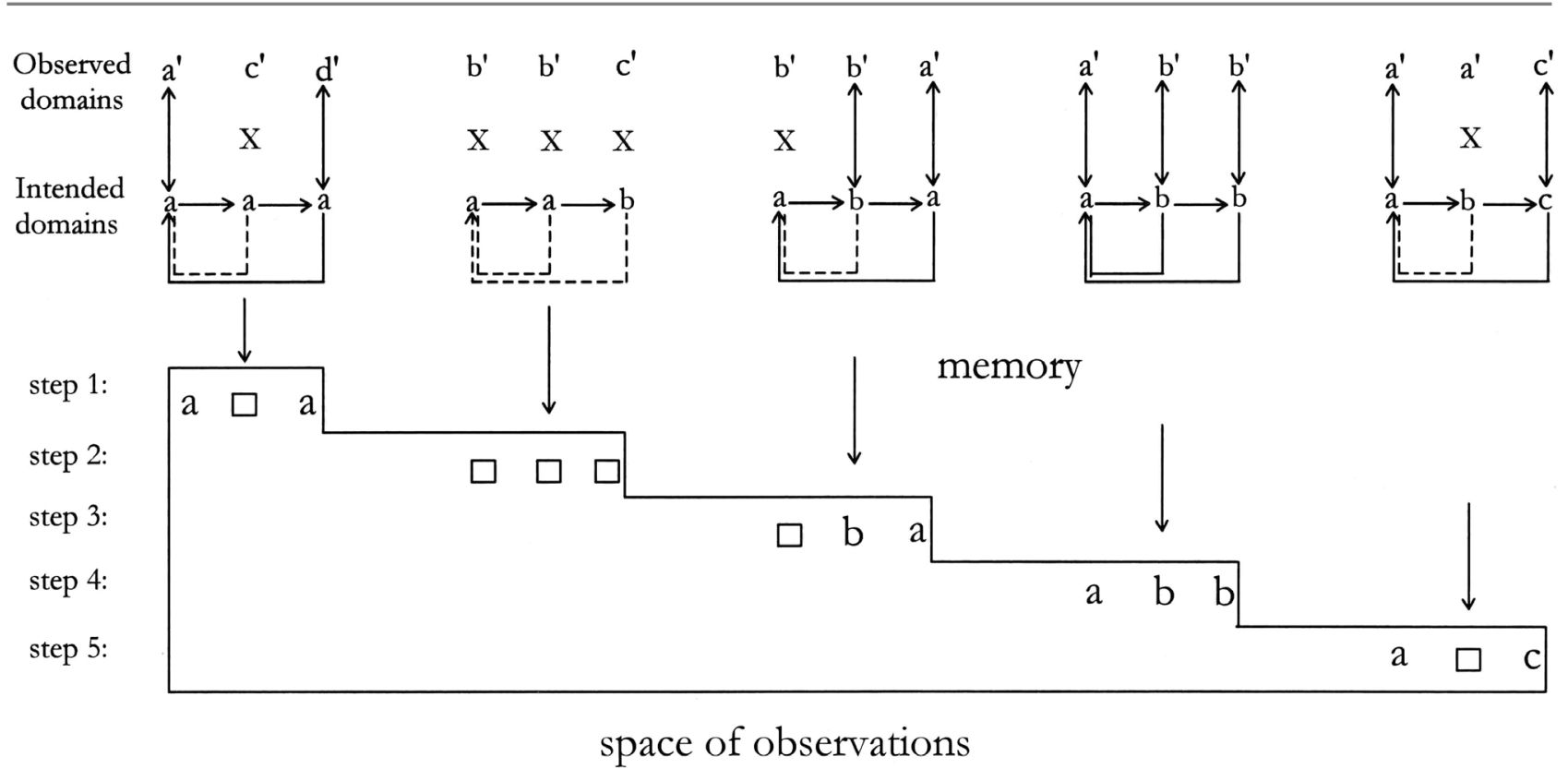

Intended and observed domains represent a double cycle-place structure generating a match/mismatch memory of intended domains of observation in the environment. The tritograms $(n=3)$ represent receptor places $(a, b, c)$. The place structure (tritograms) of the intended domains builds five receptor complexes. The receptor places of the observed domains are randomly chosen. The first intended domain [a] corresponds to the observed domain [a']. The comparison between both domains is designated by a double headed arrow in the sense of a match. Counting forward to the next intended domain [a] a non-correspondence with the observed domain [b'] is designated by a cross. Counting back to the first place [a] a cycle cannot be generated (dotted line), which is stored in memory as blank $[\square]$. The counting process from the first place- or receptor complex to the fifth receptor complex generates matches and mismatches stored in memory. Note that the stepwise comparisons of intended observations with observations in the environment generate a space of observations stored in memory.

Figure 5. Intentional self-observing operations.

inappropriate (cross). Decisively, by counting back from [a'] to [a], a cycle cannot be generated (dotted line) which is stored in memory as blank ( $\square)$. On the third position the intended quality [a] corresponds to the observed quality [a'] so that a cycle can be generated. The matches and the mismatches are stored in memory. This principle of self-observation is shown in all five complexes of the system. Note that the observed domains are randomly chosen. Since the operations of intended observations in the environment are based on the cyclic organization by proemial counting, all match/mismatch operations run self-reflexively.

If the robot has stepwise explored the environment, it must exactly go back to the route of the observed intended domains. This implies that the mismatch of domains must be ignored. In my perspective, a subjective behavior is only generated if a system is equipped with intentions that structure information consistent with the logic of acceptance and rejection [16] [61] [62]. According to Guenther [16] the capability of rejection is an index of subjectivity. Hence, on the way back to the starting point the robot must ignore (reject) domains in the environment that are inappropriate to its intentional programs. Now, the generated space of intended and experienced observations stored in memory represents the exact locations for carrying out specific operations.

Figure 6 outlines an intentional self-observing computer system. The intended observation computer activates the perception computer (1). The activation 


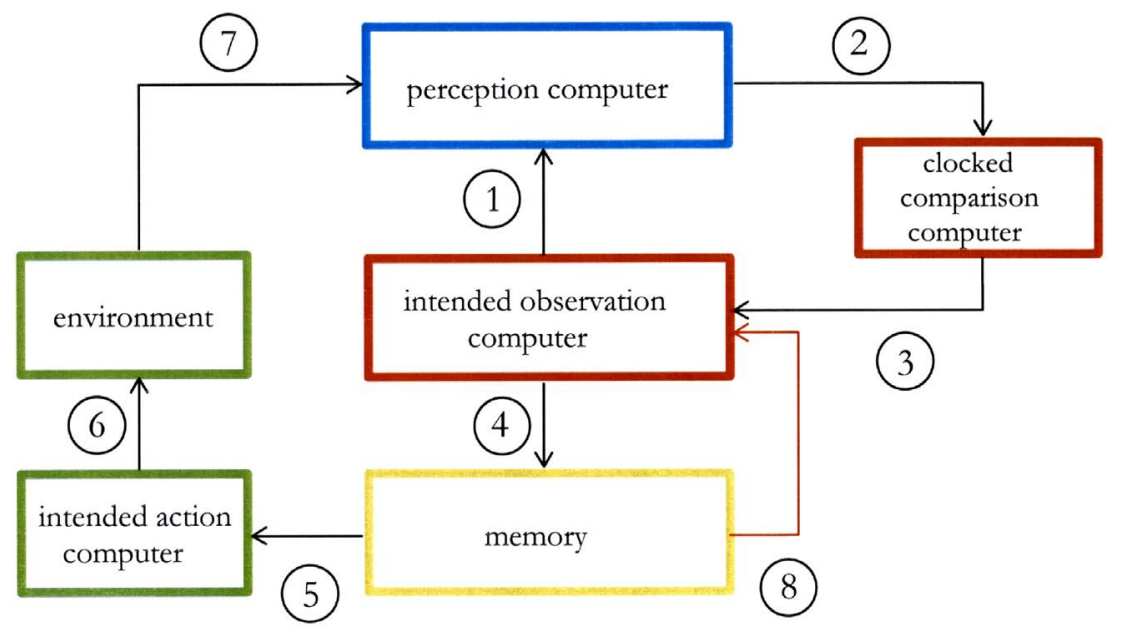

The intended observation computer activates the perception computer (1). The activation pattern of observed domains activates the clocked comparison cycle (2) that compares observed domains with the intended domains of observation (3). The pattern of observations is stored in memory (4), activating the intended action computer (5), processing operations in the environment (6). Changing environmental information is processed in the perception computer (7) and self-observed in the clocked comparison cycle. The memory can activate (red arrow) a new program of intended observation. The whole computer system runs in cycles.

Figure 6. Diagram of the intentional self-observing computer system.

pattern of observed domains activates the clocked comparison cycle (2) that compares the observed domains with the intended domains of observation (3). Matched and mismatched domains are stored in memory (4). The pattern of observations stored in memory represents a space of observations that commands (5) where the intended action computer must operate in the environment (6). Changing environmental information is processed in the perception computer (7) and self-observed again in the clocked comparison cycle. After a number of cycles of self-observation in the environment, the memory can activate (red arrow) a new program of intended observations, either programmed by the modeler or environment-dependent. As depicted in Figure 6, the whole computer system runs in cycles. Regarding the formation of memory, Pereira and coworkers [2013] demonstrated in a "calcium wave model" of tripartite synapses how astroglial modulation of neuronal activity only exerts a positive feedback by relevant information contents, but not by irrelevant ones.

Note that in this computer system memory exerts a double function: as memory and as an intentional program for further operations [62]. Admittedly, the present study does not attempt to deal with technical details, although this has partly been done with regard to an intentional computer [63] and a clocked perception system [64]. Basically, the intentional self-observing system could operate "at the bottom" so that the robot may be self-conscious in various operations.

\section{Concluding Remarks}

The model of self-observation outlined focuses on cyclic astrocyte-synapse interactions and the mechanism of receptor-receptor coupling in tripartite synapses. 
In those receptor-receptor complexes the act of self-observation is generated if the actual environmental information is appropriate to the intended domain of observation and is experienced as "its own". It is suggested that this biophysical correlate of self-observation can be implemented in a robot brain. In exploring an unknown environment the robot may be able to stepwise construct an observational space commanded and controlled by intended actions, self-referring matches and mismatches between intended observations and the environmental information, based on a cyclic organization of the mechanism.

Approaches to implement brain-inspired architectures for autonomous agents are faced with the problem of adapting behavioral needs not anticipated by the human modeler [65] [66]. Importantly, Haikonen [67] showed that a mismatch between behavioral outcome according to current sensory input signals and the expected outcome based on previously learned internal models triggers an adaptation of object and situation models. Moreover, Damerov and coworkers [66] developed a model of self-referential autonomous learning applying concepts similar to those in my proposed model [68]. Accordingly, a situation is rather the task-driven interpretation of a scene referring also to behavioral models, action outcomes, and internal states of the subject, such as intentions and goals [69] [70]. This approach to autonomous agents is based on feedback mechanisms. Importantly, both feedback expectation and expectation specialization are applied in explaining and predicting external feedback. If a different feedback instead of the expected occurs, expectation specialization is performed [66]. The concept of feedback expectation may correspond to feedforward mechanisms in astrocyte domains and their tripartite synapses.

In the perspective of the presented model the architecture of self-conscious machines elaborated by Chella and Gaglio [71] is of special importance. They implemented a robot cognitive architecture with sub-conceptual, conceptual, iconic, and linguistic areas generating artificial qualia in machines. In this architecture the higher-order of perception of the robot is the basis for self-consciousness. Here, self-consciousness has been considered a type of consciousness in general [54]. Basically, the concept of "Self" is a fundamental characteristic of living systems with first person consciousness [15] [46] [59]. Therefore, I suggest that preferred locations of self-observation in the human brain could mediate selfobserving cellular systems and the implementation of robots with consciousness. Cyclically organized astrocyte-synapse interactions forming synaptic and extrasynaptic receptor-receptor complexes may provide a candidate system.

\section{Acknowledgements}

I am very indebted to Birgitta Kofler-Westergren for preparing the final version of this paper.

\section{References}

[1] Seifert, G. and Steinhäuser, C. (2001) Ionotropic Glutamate Receptors in Astrocytes. 
Progress in Brain Research, 132, 287-299.

https://doi.org/10.1016/S0079-6123(01)32083-6

[2] Perea, G., Sur, M. and Araque, A. (2014) Neuron-Glia Networks: Integral Gear of Brain Function. Frontiers in Cellular Neuroscience, 8, 378.

https://doi.org/10.3389/fncel.2014.00378

[3] Araque, A., Parpura, V., Sanzgiri, R.P. and Haydon, P.G. (1999) Tripartite Synapses: Glia, the Unacknowledged Partner. Trends in Neurosciences, 22, 208-215. https://doi.org/10.1016/S0166-2236(98)01349-6

[4] Verkhratsky, A. and Butt, A. (2013) Glial Physiology and Pathophysiology. Wiley, West Sussex. https://doi.org/10.1002/9781118402061

[5] Tononi, G. and Koch, C. (2015) Consciousness: Here, There, and Everywhere? Philosophical Transactions of the Royal Society of London, Series B, Biological Science, 370, 20140167.

[6] Mitterauer, B.J. (2013) Robots with Consciousness: Creating a Third Nature. InternationalJournal of Machine Consciousness, 5, 179-193.

https://doi.org/10.1142/S1793843013500054

[7] Reggia, J.A. (2013) The Rise of Machine Consciousness: Studying Consciousness with Computational Models. Neural Networks, 44, 112-131.

https://doi.org/10.1016/j.neunet.2013.03.011

[8] Manzotti, R. (2013) Machine Consciousness: A Model Approach. Natural Intelligence, 2, 7-18.

[9] Chella, A. and Manzotti, R. (2014) Robot Consciousness: Theoretical and Empirical Issues. The $13^{\text {th }}$ International Conference on Intelligent Autonomous Systems, Padova, 15-19 July 2014.

[10] Cheng, G. (2015) Humanoid Robotics and Neuroscience: Science, Engineering and Society. Frontiers in Neuroengineering Series. CRC Press/Taylor and Francis, Boca Raton.

[11] Schulz, H., Ott, L., Sturm, J. and Burgard, W. (2009) Learning Kinematics from Direct Self-Observation using Nearest-Neighbor Methods. Proceedings of the German Workshop on Robotics, Braunschweig.

[12] Bohannon, J. (2014) Helping Robots See the Big Picture. Science, 346, 186-187. https://doi.org/10.1126/science.346.6206.186

[13] Quinonez, V., Ramirez, M., Lizarraga, C., Tostado, I. and Bakios, J. (2015) Autonomous Robot Navigation Based Pattern Recognition Techniques and Artificial Neural Networks. In: Ferrandez, V.J., et al., Eds., Bioinspired Computation in Artificial Systems, Lecture Notes in Computer Science, Vol. 9108, Springer, Cham.

[14] Fuxe, K., Agnati, L.F., Marcoli, M. and Borroto-Escuela, D.O. (2015) Volume Transmission in Central Dopamine and Noradrenaline Neurons and Its Astroglial Targets. Neurochemical Research, 40, 2600-2614. https://doi.org/10.1007/s11064-015-1574-5

[15] Mitterauer, B.J. (2017) The Astrocyte as a Mediator for Self-Reflexive Agents. Advances in Bioscience and Biotechnology, 8, 195-210. https://doi.org/10.4236/abb.2017.86015

[16] Guenther, G. (1962) Cybernetic Ontology and Transjunctional Operations. In: Yovits, M.C., et al., Eds., Self-Organizing Systems, Spartan Books, Washington DC, 313-392.

[17] Mitterauer, B.J. (2013) The Proemial Synapse: Consciousness-Generating Glial-Neuronal Units. In: Pereira, A. and Lehmann, D., Eds., The Unity of Mind, Brain 
and World, Cambridge University Press, Cambridge, 233-264. https://doi.org/10.1017/CBO9781139207065.009

[18] Nedergard, M., Ransom, B. and Goldman, S.A. (2003) New Roles for Astrocytes: Redefining the Functional Architecture of the Brain. Trends in Neurosciences, 26, 523-530. https://doi.org/10.1016/j.tins.2003.08.008

[19] Verkhratsky, A. and Nedergaard, M. (2014) Astroglial Cradle in the Life of the Synapse. Philosophical Transactions of the Royal Society B, 369, Article ID: 20130595. https://doi.org/10.1098/rstb.2013.0595

[20] Kimelberg, H.K. (2010) Functions of Mature Mammalian Astrocytes: A Current View. Neuroscientist, 16, 79-106. https://doi.org/10.1177/1073858409342593

[21] Lavialle, M., Aumann, G., Anlauf, E., Pröls, F., et al. (2011) Structural Plasticity of Perisynaptic Astrocyte Processes Involves Ezrin and Metabotropic Glutamate Receptors. Proceedings of the National Academy of Sciences, 108, 12915-12919. https://doi.org/10.1073/pnas.1100957108

[22] Eroglu, C. and Barres, B.A. (2010) Regulation of Synaptic Connectivity by Glia. Nature, 468, 223-231. https://doi.org/10.1038/nature09612

[23] DePitta, M., Volman, V., Berry, H., Parpura, V., et al. (2012) Computational Quest for Understanding the Role of Astrocyte Signaling in Synaptic Transmission and Plasticity. Frontiers in Computational Neuroscience, 6, 98.

[24] DePitta, M., Brunel, N. and Volterra, A. (2016) Astrocytes: Orchestrating Synaptic Plasticity. Neuroscience, 323, 43-61. https://doi.org/10.1016/j.neuroscience.2015.04.001

[25] Kettenmann, H. and Zorec, R. (2013) Release of Gliotransmitters and Transmitter Receptors in Astrocytes. In: Kettenmann, H. and Ransom, B.R., Eds., Neuroglia, Oxford University Press, New York, 197-211.

[26] Bradley, S.J. and Challiss, J. (2012) G Protein-Coupled Receptor Signalling in Astrocytes in Health and Disease: A Focus on Metabotropic Glutamate Receptors. Biochemical Pharmacology, 84, 249-259. https://doi.org/10.1016/j.bcp.2012.04.009

[27] Porto-Pazos, A.B., Veiguela, N., Mesejo, P., Navarrate, M., et al. (2011) Artificial Astrocytes Improve Neural Network Performance. PLoS ONE, 6, e19109. https://doi.org/10.1371/journal.pone.0019109

[28] Mitterauer, B.J. (2012) Qualitative Information Processing in Tripartite Synapses: A Hypothetical Model. Cognitive Computation, 4, 181-194.

https://doi.org/10.1007/s12559-011-9115-2

[29] Min, R., Santello, M. and Nevian, T. (2012) The Computational Power of Astrocyte Mediated Synaptic Plasticity. Frontiers in Computational Neuroscience, 6, 93. https://doi.org/10.3389/fncom.2012.00093

[30] Kozachkov, L. and Michmizos, K.P. (2017) The Causal Role of Astrocytes in Slow-Wave Rhythmogenesis: A Computational Modelling Study.

[31] Volman, V., Bazhenov, M. and Sejnowsky, J. (2012) Computational Models of Neuron-Astrocyte Interaction in Epilepsy. Frontiers in Computational Neuroscience, 6, 58. https://doi.org/10.3389/fncom.2012.00058

[32] Nadkarni, S., Jung, P. and Levine, H. (2008) Astrocytes Optimize the Synaptic Transmission of Information. PLOS Computational Biology, 4, e1000088. https://doi.org/10.1371/journal.pcbi.1000088

[33] DiCastro, M.A., Chuquet, J., Liaudet, N., Bhaukaurally, K., et al. (2011) Local Ca ${ }^{2+}$ Detection and Modulation of Synaptic Release by Astrocytes. Nature Neuroscience, 14, 1276-1284. https://doi.org/10.1038/nn.2929 
[34] Araque, A., Carmignoto, G., Haydon, P.G., Oliet, S.H.R., et al. (2014) Gliotransmitters Travel in Time and Space, Neuron, 81, 728-739. https://doi.org/10.1016/j.neuron.2014.02.007

[35] Kimelberg, H.K. (2012) Protoplasmic Mammalian Astrocytes. Morphology, Interrelationships and Implications for Function. In: Scemes, E. and Spray, C., Eds., $A s$ trocytes Wiring the Brain, Frontiers in Neuroscience, Vol. 1, Boca Raton, 3-24.

[36] Baer, W. and Mitterauer, B.J. (2015) Der Körper, Geist und Seele in der Ereignis-orientierten Weltanschauung. Grundlagenstudien aus Kybernetik und Geisteswissenschaft, 56, 3-20.

[37] Halassa, M.M., Fellin, T. and Haydon, P.G. (2007) The Tripartite Synapse: Roles for Gliotransmission in Health and Disease. Trends in Molecular Medicine, 13, 54-63. https://doi.org/10.1016/j.molmed.2006.12.005

[38] Oliet, S.H.R. and Mothet, J.P. (2006) Molecular Determinants for D-Serine-Mediated Gliotransmission: From Release to Function. Glia, 54, 726-737. https://doi.org/10.1002/glia.20356

[39] Zhang, Q. and Haydon, P.G. (2005) Roles for Gliotransmission in the Nervous System. Journal of Neural Transmission, 112, 121-125. https://doi.org/10.1007/s00702-004-0119-x

[40] Harada, K., Taichi, K. and Takashi, T. (2016) Gliotransmitter Release from Astrocytes: Functional, Developmental, and Pathological Implications in the Brain. Frontiers in Neuroscience, 9, 499. https://doi.org/10.3389/fnins.2015.00499

[41] Fellin, T., Pascual, O., Gobbo, S., Pozzan, T., et al. (2004) Neuronal Synchrony Mediated by Astrocytic Glutamate through Activation of Extrasynaptic NMDA Receptors. Neuron, 43, 729-743. https://doi.org/10.1016/j.neuron.2004.08.011

[42] Mothet, J.P., Pollegioni, L., Ouanounou, G., Martineau, M., Fossier, P. and Baux, G. (2005) Glutamate Receptor Activation Triggers a Calcium-Dependent and SNARE Protein-Dependent Release of the Gliotransmitter D-Serine. Proceedings of the National Academy of Sciences, 102, 5606-5611. https://doi.org/10.1073/pnas.0408483102

[43] Van Horn, M.R., Sild, M. and Ruthazer, E.S. (2013) D-Serine as a Gliotransmitter and Its Roles in Brain Development and Disease. Frontiers in Cellular Neuroscience, 7, 39. https://doi.org/10.3389/fncel.2013.00039

[44] Newman, E.A. (2003) New Roles for Astrocytes: Regulation of Synaptic Transmission. Trends in Neurosciences, 26, 536-542. https://doi.org/10.1016/S0166-2236(03)00237-6

[45] Guenther, G. (1976) Cognition and Volition. A Contribution to a Theory of Subjectivity. In: Kanitscheider, B., Ed., Sprache und Erkenntnis, Festschriftfür Gerhard Frey, AMOE, Innsbruck.

[46] Baer, W. (2017) Does the Rose-Tinted Glassed Effect in Contemporary Physics Prevent Us from Explaining Consciousness? Journal of Consciousness Studies, 24, 7-8.

[47] Borroto-Escuela, D.O., Agnati, L.F., Bechter, K., Jansson, A., et al. (2015) The Role of Transmitter Diffusion and Flow versus Extracellular Vesicles in Volume Transmission in the Brain Neural-Glial Networks. Philosophical Transactions of the Royal Society of London. Series B, 370, Article ID: 20140183. https://doi.org/10.1098/rstb.2014.0183

[48] Dityatev, A., Seidenbecher, C.I. and Schachner, M. (2010) Compartmentalization from the Outside: The Extracellular Matrix and Functional Microdomains in the Brain. Trends in Neuroscience, 33, 503-512. 
https://doi.org/10.1016/j.tins.2010.08.003

[49] Fuxe, K., Borroto-Escuela, D.O., Romero-Fernandez, W., Ciruela, F., et al. (2012) On the Role of Volume Transmission and Receptor-Receptor Interactions in Social Behaviour: Focus on Central Catecholamine and Oxytocin Neurons. Brain Research, 1476, 119-131. https://doi.org/10.1016/j.brainres.2012.01.062

[50] Fuxe, K., Borroto-Escuela, D.O., Ciruela, F., Guidolin, V.D. and Agnati, L.F. (2014) Receptor-Receptor Interactions in Heteroreceptor Complexes: A New Principle in Biology. Focus on Their Role in Learning and Memory. Neuroscience Discovery, 2, 6. https://doi.org/10.7243/2052-6946-2-6

[51] Tarakanov, A.O. and Fuxe, K.G. (2010) Triplet Puzzle: Homologies of Receptor Heteromers. Journal of Molecular Neuroscience, 42, 294-303. https://doi.org/10.1007/s12031-009-9313-5

[52] Schel, M.A., Kühn, S., Brass, M., Haggard, P., et al. (2014) Neural Correlates of Intentional and Stimulus-Driven Inhibition: A Comparison. Frontiers in Human Neuroscience, 8, 27. https://doi.org/10.3389/fnhum.2014.00027

[53] Proekt, A., Brezina, V. and Weiss, K.R. (2004) Dynamical Basis of Intentions and Expectations in a Simple Neuronal Network. Proceedings of the National Academy of Sciences, 101, 9447-9452. https://doi.org/10.1073/pnas.0402002101

[54] Boyles, R.J. (2012) Artificial Qualia, Intentional Systems and Machine Consciousness.

[55] Mitterauer, B. (2007) Where and How Could Intentional Programs Be Generated in the Brain? A Hypothetical Model Based on Glial-Neuronal Interactions. BioSystems, 88, 101-112.

[56] Wang, M., Wong, A.H. and Liu, F. (2011) Interactions between NMDA and Dopamine Receptors: A Potential Therapeutic Target. Brain Research, 1476, 154-163. https://doi.org/10.1016/j.brainres.2012.03.029

[57] Flock, T., Hauser, A.S., Lund, N., Gloriam, D.E., et al. (2017) Selectivity Determinants of GPCR-G-Protein Binding. Nature, 545, 317-322.

https://doi.org/10.1038/nature22070

[58] Mevorach, C., Hodsoll, J., Allen, H., Shalev, L. and Humphreys, G. (2010) Ignoring the Elephant in the Room: A Neural Circuit to Downregulate Salience. Journal of Neuroscience, 30, 6072-6079. https://doi.org/10.1523/JNEUROSCI.0241-10.2010

[59] Mitterauer, B. (1998) An Interdisciplinary Approach towards a Theory of Consciousness. BioSystems, 45, 99-121.

[60] Peelen, M.V. and Downing, P.E. (2017) Category Selectivity in Human Visualcortex: Beyond Visual Object Recognition. Neuropsychologia, 105, 177-183. https://doi.org/10.1016/j.neuropsychologia.2017.03.033

[61] Mitterauer, B. (2000) Some Principles for Conscious Robots. JIS, 10, 27-57. https://doi.org/10.1515/JISYS.2000.10.1.27

[62] Mitterauer, B. (2011) Brain-Based Elementary Auto-Reflection Mechanisms for Conscious Robots. Some Philosophical Implications. International Journal of Machine Consciousness, 3, 283-308. https://doi.org/10.1142/S1793843011000820

[63] Mitterauer, B. (1988) Computer System for Simulating Reticular Formation Operation. United States Patent 4, 783, 741.

[64] Mitterauer, B. (2004) Computer System, Particularly for Simulation of Human Perception via Sense Organs. United States Patent 5, 697, 792, B2.

[65] Pratihar, D.K. and Jain, L.C. (2010) Intelligent Autonomous Systems. Springer, 
Berlin. https://doi.org/10.1007/978-3-642-11676-6

[66] Damerow, F., Knoblauch, A., Körner, U., et al. (2016) Toward Self-Referential Autonomous Learning of Object and Situation Models. Cognitive Computation, 8, 703-719. https://doi.org/10.1007/s12559-016-9407-7

[67] Haikonen, P.O.A. (2014) Yes and No: Match/Mismatch Function in Cognitive Robots. Cognitive Computation, 6, 158-163. https://doi.org/10.1007/s12559-013-9234-Z

[68] Koerner, E., Knoblauch, A. and Koerner, U. (2015) Autonomous Situation Understanding Self-Referential Learning of Situation Representations in a Brain-Inspired Architecture. In: Advances in Cognitive Neurodynamics (IV): Proceedings of the Fourth International Conference on Cognitive Neuro-Dynamics, Springer, Dordrecht, 497-501.

[69] Pfalzgraf, J. and Mitterauer, B. (2005) Towards a Biomathematical Model of Intentional Multiagent Systems. In: Diaz, A., et al., Eds., Eurocast, LNCS 3642, Springer, Berlin, 577-583.

[70] Manzotti, R. and Jeschko, S. (2014) From the Perspective of Artificial Intelligence: A New Approach to the Nature of Consciousness. International Journal of Advanced Research in Artificial Intelligence, 3, 1-12. https://doi.org/10.14569/IJARAI.2014.031201

[71] Chella, A. and Gaglio, S. (2012) Synthetic Phenomenology and High-Dimensional Buffer Hypothesis. International Journal of Machine Consciousness, 4, 353-365. https://doi.org/10.1142/S1793843012400203 


\section{Abbreviations}

Ac: Astrocyte

asr: Astrocytic receptor

AMPR: Alpha-amino-3-hydroxy-5-methyl-4-isoxalone-proprionoc acid ATP: Adenosine-tri-phosphate

$\mathrm{Ca}^{2+}:$ Calcium

ECM: Extracellular matrix

esr: Extrasynaptic receptor

GLU: Glutamate

GPCRs: G-protein coupled receptors

GT: Gliotransmitter

NA: Noradrenaline

NMDA: N-methyl-D-asparate

nSy: Neuronale synapse

por: Postsynaptic receptor

PSAPs: Perisynaptic astrocytic processes

psr: Postsynaptic receptor

PVAs: Perivascular astrocytic processes

SE: Serotonine

Sy: Synapse 